\section{Relationship between the phenylephrine test and eyelid droop after aponeurotic repair with the use of an epinephrine-containing local anaesthetic}

H Matsuda', T Shiba², Y Takahashi ${ }^{3}$

and $\mathrm{H}$ Tsuneoka ${ }^{2}$

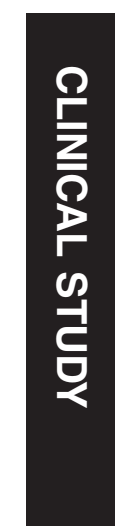

phenylephrine response and the $\triangle \mathrm{MRD}-1$ overall. This indicates that the degree of postoperative eyelid droop can be estimated by the phenylephrine test results in transcutaneous aponeurotic repair.

Eye (2018) 32, 93-98; doi:10.1038/eye.2017.153; published online 4 August 2017

\section{Introduction}

Obtaining optimal symmetrical eyelid height after blepharoptosis surgery is challenging, ${ }^{1}$ and it is essential for oculoplastic surgeons to identify factors affecting postoperative eyelid height. ${ }^{2,3}$ Intraoperative quantification is usually used to determine appropriate advancement of the levator aponeurosis during the transcutaneous approach. ${ }^{1}$ However, epinephrine-containing local anaesthetic stimulates the Müller muscle for several hours, ${ }^{4}$ which occasionally results in less advancement of the levator aponeurosis during surgery, resulting in an undercorrected upper eyelid position after loss of the epinephrine effect. 5,6 The frequency and degree of the upper eyelid droop after loss of the epinephrine effect has not been examined.

Application of topical phenylephrine hydrochloride stimulates the Müller muscle and subsequently raises the upper eyelid. ${ }^{7}$ As the degree of this response reflects Müller muscle function, a preoperative phenylephrine test is routinely performed to determine the appropriate amount of Müller muscle conjunctival resection (MMCR). ${ }^{8}$ We assumed that the results of the phenylephrine test were applicable to preoperative prediction of the upper eyelid droop after loss of the epinephrine effect.

\author{
${ }^{1}$ Department of \\ Ophthalmology, Daisan \\ Hospital, The Jikei \\ University School of \\ Medicine, Komae, Tokyo, \\ Japan
}

${ }^{2}$ Department of Ophthalmology, The Jikei University School of Medicine, Tokyo, Japan

${ }^{3}$ Department of Oculoplastic, Orbital and Lacrimal Surgery, Aichi Medical University Hospital, Nagakute, Aichi, Japan

Correspondence: H Matsuda, Department of Ophthalmology, Daisan Hospital, The Jikei

University School of Medicine, 4-11-1, Izumihonchou, Komae, Tokyo 201-8601, Japan Tel: +8133480 1151 (ext. 3241);

Fax: +8133480 3611

E-mail: thkmmm@yahoo.co.jp

Received: 19 December 2016

Accepted in revised form: 19 June 2017 Published online: 4 August 2017 
In the following study, we examined the relationship between the results of the phenylephrine test and the degree of postoperative upper eyelid droop after transcutaneous aponeurotic repair.

\section{Patients and methods}

This was a retrospective review of the data from all patients who underwent transcutaneous aponeurotic repair for aponeurotic blepharoptosis performed by one oculoplastic surgeon (HM) between April 2014 and March 2016. Patients with a history of upper eyelid surgery, levator function $<5 \mathrm{~mm}$, and a follow-up period $<3$ months were excluded from the study. Patients who underwent simultaneous removal of redundant skin were also excluded, as this procedure required a larger volume of injected local anaesthetic than ptosis surgery without skin removal, which caused intraoperative mechanical ptosis and affected the intraoperative quantification.

Institutional Review Board (IRB) approval was obtained from The Jikei Medical University (number 27-321), and the protocol adhered to the tenets of the Declaration of Helsinki. As this was not an interventional study, the IRB granted a waiver of a written informed consent for this study on the basis of the ethical guidelines for medical and health research involving human subjects established by the Japanese Ministry of Education, Culture, Sports, Science and Technology, and by the Ministry of Health, Labour and Welfare. Nevertheless, the IRB requested us to present an outline description of this study to the public via a notice board in our institution to provide an additional opportunity for patients to refuse participation in this study, before patient records were deidentified and made anonymous. None of the patients declined participation.

The following data were collected: patient age, sex, surgical side, results of the phenylephrine test, volume of local anaesthetic injected, margin reflex distance (MRD)-1, levator function, and postoperative complications. All examinations were performed by one of the authors (HM). MRD-1 was measured before, during, and 3 months after surgery. MRD-1 was determined as the distance from the upper eyelid margin to the corneal light reflex in the primary eye position. The distance was measured using a mm ruler while the patient was in the sitting position and looking at a light source (a penlight). ${ }^{9}$ All measurements of the eyelid height were measured in units of $0.5 \mathrm{~mm}$. An intraoperative measurement was performed immediately before creation of the eyelid crease. The $\triangle \mathrm{MRD}-1$ was defined as the difference between the postoperative MRD-1 value and the intraoperative value.
A 5\% phenylephrine test was preoperatively performed on the surgical side. A positive response was defined as an increase in MRD- 1 of $\geq 0.5 \mathrm{~mm} 20 \mathrm{~min}$ after application of the phenylephrine eye drop. ${ }^{10}$ We confirmed the secure application of the phenylephrine eye drop by a dilated pupil measurement.

Patients were classified into two groups according to the results of phenylephrine test as Group A (positive response) and Group B (negative response).

The patient age and the measurement values were expressed as the mean values \pm standard deviation. Intergroup differences in patient age, volume of local anaesthetic, and levator function were examined using the Mann-Whitney $U$ test. The male-to-female ratio and the ratio of the surgical side were compared between the groups using the $X^{2}$ test for independent variables. The comparison of the mean pre- and intraoperative MRD-1s was performed between the groups using the MannWhitney $U$ Test. The Wilcoxon signed-rank test was used to compare the intra- and postoperative MRD-1s in the total patient group, Group A, and Group B, as the populations were not normally distributed. The $\triangle \mathrm{MRD}-1$ was compared between the groups using the MannWhitney $U$ test.

Patient age, sex, pre- and intraoperative MRD-1s, levator function, and phenylephrine response were investigated as possibly influencing the $\triangle$ MRD-1. Patient sex was expressed using a binary system (a dummy variable; $0=$ male, $1=$ female). The relationship between the influential factors and $\triangle \mathrm{MRD}-1$ was analysed using single and subsequent multiple regression analysis in the total group and in Group A. We obtained a statistical error of the relationship in Group B, as the results of phenylephrine tests were 0 in all patients in Group B.

The statistical significance for each analysis was defined as $P<0.05$. All analyses were performed using JMP version 12 software (SAS, Cary, NC, USA).

\section{Surgical technique}

A skin incision line 20-22 mm long was marked. We usually set an incision line $7 \mathrm{~mm}$ above the eyelid margin; however, in patients with a mild amount of redundant skin, we set a high incision line $(8-9 \mathrm{~mm}$ above the eyelid margin) to prevent excess skin hooding from the eyelid crease. Local anaesthetic of $1 \%$ buffered lidocaine and a 1 : 100000 dilution of epinephrine without hyaluronidase was injected subcutaneously around the skin incision line. A skin incision was made using a number 15 blade. The layer under the orbicularis oculi muscle was dissected to expose the tarsal plate. The posterior lamella of the levator aponeurosis, which extends to the tarsal plate, ${ }^{11}$ was incised at its attachment site and dissected from the tarsal plate with Westcott scissors until the insertion of the 
Table 1 Summary of the patient data, measurement results, and statistical comparisons

\begin{tabular}{lcccc}
\hline & & P & \\
\cline { 2 - 5 } & Total & Group A & Group B & Group A vs B \\
\cline { 2 - 5 } No. of eyelids/patients & $66 / 40$ & $26 / 16$ & $40 / 24$ & - \\
No. of patients bilateral/unilateral & $26 / 14$ & $10 / 6$ & $22 / 18$ & $0.525^{\mathrm{a}}$ \\
Right/left & $34 / 32$ & $13 / 13$ & $6 / 18$ & $0.953^{\mathrm{a}}$ \\
Male/female & $14 / 26$ & $8 / 8$ & $0.542^{\mathrm{a}}$ \\
Age (range), yrs & $70.6 \pm 9.4(47$ to 87$)$ & $68.5 \pm 9.0(54$ to 87$)$ & $70.8 \pm 9.1(47$ to 87$)$ & $0.589^{\mathrm{b}}$ \\
Levator function (range), mm & $13.8 \pm 4.5(6.5$ to 16$)$ & $13.2 \pm 4.5(8.0$ to 15.5$)$ & $13.9 \pm 4.6(6.5$ to 16$)$ & $0.835^{\mathrm{b}}$ \\
Volume of anaesthetics (range), mm & $0.75 \pm 0.28(0.6$ to 1.6$)$ & $0.80 \pm 0.35(0.6$ to 1.5$)$ & $0.73 \pm 0.25(0.7$ to 1.6$)$ & $0.899^{\mathrm{b}}$ \\
Preoperative MRD-1 (range), mm & $0.06 \pm 0.81(-2.0$ to 2.5$)$ & $0.09 \pm 1.17(-1.5$ to 2.5$)$ & $0.04 \pm 0.58(-2.0$ to 2.0$)$ & $0.669^{\mathrm{b}}$ \\
MRD-1 using phenylephrine eye drop & $0.55 \pm 1.01(-2.0$ to 3.0$)$ & $1.22 \pm 1.21(-0.5$ to 3.0$)$ & $0.04 \pm 0.58(-2.0$ to 2.0$)$ & $<0.001^{\mathrm{c}}$ \\
Intraoperative MRD-1 (range), mm & $2.46 \pm 1.11(2.0$ to 4.5$)$ & $2.80 \pm 0.79(2.0$ to 4.5$)$ & $2.30 \pm 1.03(2.0$ to 4.0$)$ & $0.225^{\mathrm{b}}$ \\
Postoperative 3-month MRD-1 (range), mm & $2.12 \pm 1.0(0$ to 4.5$)$ & $2.12 \pm 1.05(0$ to 4.5$)$ & $2.13 \pm 1.01(0.5$ to 4.0$)$ & $0.915^{\mathrm{b}}$ \\
MMRD-1 (range), mm & $0.34 \pm 0.55(0$ to 2.0$)$ & $0.68 \pm 0.52(0$ to 2.0$)$ & $0.17 \pm 0.56(0$ to 1.5$)$ & $0.004^{\mathrm{c}}$ \\
P-value: intra vs post 3-month MRD-1 & $<0.001^{\mathrm{d}}$ & $<0.001^{\mathrm{d}}$ & $0.045^{\mathrm{d}}$ & - \\
\hline
\end{tabular}

Group A: positive phenylephrine response group (16 patients), Group B: negative phenylephrine response group (24 patients), MRD: margin reflex distance.

The $\triangle$ MRD-1 was the difference between the 3-month postoperative MRD-1 value and the intraoperative value. ${ }^{a}$ No statistical significance using the $X^{2}$ test. ${ }^{\mathrm{b}}$ No statistical significance using the Mann-Whitney $U$ test. ${ }^{\mathrm{c}}$ Statistical significance using the Mann-Whitney $U$ test. ${ }^{\mathrm{d}}$ Statistical significance using the Wilcoxon signed-rank test.

Müller muscle onto the upper edge of the tarsal plate was exposed. The levator aponeurosis was easily bluntly dissected away from the Müller muscle using a cotton swab, as the posterior lamella does not firmly attach to the Müller muscle. ${ }^{11}$ The orbital septum was then incised transversely to expose the anterior lamella of the levator aponeurosis, which joins the orbital septum. ${ }^{11}$ The levator aponeurosis was advanced and secured to the upper onethird of the tarsal plate with a 6-0 Asflex suture (Kono Seisakusho, Tokyo, Japan). The advancement was repeated until an adequate eyelid height was obtained. If necessary, the levator aponeurosis was fixed to the tarsal plate at one or two additional points to create a natural curvature. At this time, the intraoperative MRD-1 was measured in the sitting position. Finally, an eyelid crease was created at three points using 6-0 Asflex buried sutures, and the wound was closed with 6-0 Asflex sutures.

Postoperative medications consisted of oral levofloxacin for 3 days, and $0.3 \%$ ofloxacin ointment and $0.5 \%$ topical levofloxacin for 2 weeks.

\section{Results}

Patient data, measurement results, and statistical comparisons are shown in Table 1. Although 58 patients underwent blepharoptosis surgery, 18 patients were excluded because of prior blepharoptosis surgery in two patients, poor levator function in two patients, an insufficient follow-up period in three patients, and simultaneous blepharoplasty in 11 patients. This study included a final total of 66 eyelids (34 right, 32 left) in 40 patients (14 males, 26 females; mean age, 70.6 years; range, 47-87 years). Group A comprised 26 eyelids in 16 patients and Group B comprised 40 eyelids in 24 patients. Bilateral surgery was performed in 26 patients, 10 of whom were in Group A and 16 were in Group B. The mean follow-up period was 6 months (range, 4-13 months). There was no significant difference between the groups in patient age, male-to-female ratio, surgical side, levator function, or volume of injected local anaesthetic (all $P>0.05$ ).

There was no significant difference between the groups in mean preoperative MRD-1 (Group A, 0.09 $\pm 1.17 \mathrm{~mm}$; Group B, $0.04 \pm 0.58 \mathrm{~mm} ; P=0.669)$ and intraoperative MRD-1 (Group A, $2.80 \pm 0.79$ mm; Group B, $2.30 \pm 1.03 \mathrm{~mm} ; P=0.225)$. The mean postoperative MRD-1 was significantly lower than the mean intraoperative MRD-1 in each group (total patient group, $P<0.001$; Group A, $P<0.001$; Group B, $P=0.045)$. The $\triangle \mathrm{MRD}-1$ in Group A $(0.68 \pm 0.52 \mathrm{~mm})$ was significantly greater than that in Group B $(0.17 \pm 0.56 \mathrm{~mm} ; P=0.004)$.

In the total patient group, single regression analysis showed that $\triangle \mathrm{MRD}-1$ was not significantly correlated with age $(P=0.277)$, sex $(P=0.151)$, preoperative MRD-1 $(P=0.611)$, or levator function $(P=0.755)$, while $\Delta \mathrm{MRD}-1$ was significantly correlated with intraoperative MRD-1 $(P=0.031)$ and phenylephrine test results $(P<0.001)$.

However, multiple regression analysis showed that only the phenylephrine test results had a moderate correlation with $\triangle$ MRD-1 (phenylephrine test, $P=0.002$; intraoperative MRD-1, $P=0.112 ; \mathrm{Y}_{\triangle \mathrm{MRD}-1}=0.441$ $X_{\text {phenylephrine }}+0.358 ; r=0.462 ; r^{2}=0.213$; Figure 1 ). The correlation between the presumptive influential factors and $\triangle \mathrm{MRD}-1$ did not reach statistical significance using single regression analysis in Group A $(P>0.05)$, although 


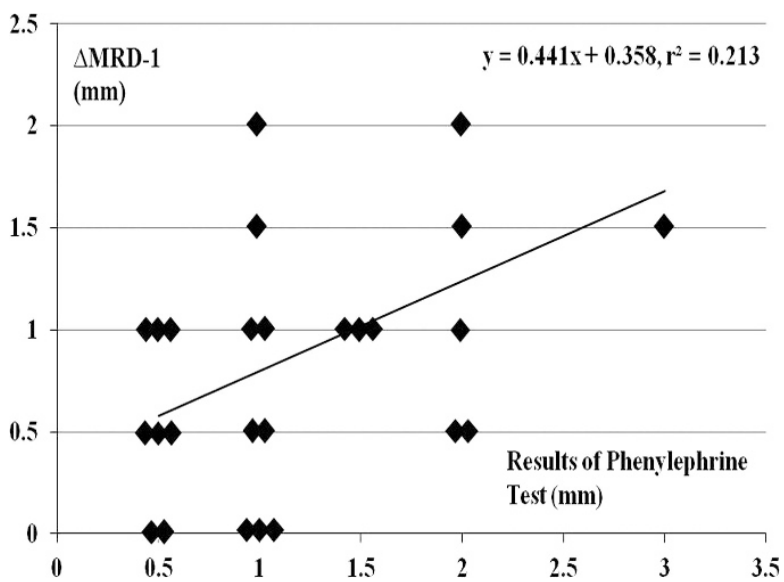

Figure 1 A scatter diagram of Group A. The results of the phenylephrine test and the $\Delta$ margin reflex distance (MRD)- 1 are shown on the $x$ and $y$ axes, respectively.

the correlation between phenylephrine response and $\triangle$ MRD-1 was close to statistical significance $(P=0.081)$.

Three eyelids (one eyelid in Group A and two eyelids in Group B) showed a mild upper eyelid oedema lasting more than 1 week, but this symptom resolved spontaneously during the measurement period. An undercorrected upper eyelid position (MRD- $1<2 \mathrm{~mm}$ ) was observed; postoperative MRD-1 was $0 \mathrm{~mm}$ in one eyelid in Group A, $0.5 \mathrm{~mm}$ in four eyelids in Group B, $1.0 \mathrm{~mm}$ in four and seven eyelids in Group A and Group $\mathrm{B}$, respectively, and $1.5 \mathrm{~mm}$ in two eyelids and five eyelids in Group A and Group B, respectively. Intereyelid height asymmetry $>1.0 \mathrm{~mm}$ was observed only in Group A: a laterality of $1.0 \mathrm{~mm}$ was shown in three patients (two were unilateral cases, and one was bilateral), $1.5 \mathrm{~mm}$ in one patient with bilateral blepharoptosis, and $2.0 \mathrm{~mm}$ in one patient with unilateral blepharoptosis. An overcorrected upper eyelid position (MRD-1 $>5.5 \mathrm{~mm}$ ) was not present in any patient during the follow-up period.

\section{Discussion}

The present study showed a relationship between the results of the phenylephrine test and postoperative upper eyelid droop. Although previous studies showed a relationship between the phenylephrine test and MMCR,, 10 we first examined the effect of the phenylephrine test on transcutaneous ptosis surgery.

The $\triangle$ MRD-1 in Group A was considerable. In addition, a significant moderately positive correlation was found between phenylephrine response and $\triangle \mathrm{MRD}-1$ in the total patient group. These results imply that although the upper eyelid droops after transcutaneous aponeurotic repair, the degree of this postoperative eyelid droop can be estimated using the results of the phenylephrine test.

In contrast, the $\triangle \mathrm{MRD}-1$ in Group B was quite small $(0.17 \mathrm{~mm})$, although the postoperative MRD-1 was significantly lower than the intraoperative MRD-1. This indicates that changes in MRD-1 after aponeurotic repair are clinically negligible in patients with negative results from the phenylephrine test.

Intraoperative MRD-1 was around $2.5 \mathrm{~mm}$ in most patients in the present study. In such patients, postoperative eyelid droop of $0.68 \mathrm{~mm}$ (Group A) largely affects quality of vision. ${ }^{12}$ Although the $\triangle \mathrm{MRD}-1$ in Group A was relatively small, we believe that the difference in $\triangle \mathrm{MRD}-1$ between the groups has clinically significant implications.

Of 66 eyelids in the present study, 23 eyelids (34.8\%) were undercorrected $(<2 \mathrm{~mm})$. This high undercorrection rate may be attributed to the intraoperative MRD-1 of $2.46 \mathrm{~mm}$. We intentionally targeted such a relatively low intraoperative MRD-1 in some patients because a high upper eyelid position results in an unsuitable appearance for typical elderly Japanese patients and occasionally worsens the condition of dry eye. ${ }^{13,14}$ Other patients had moderate levator function $(6-7 \mathrm{~mm})$ and/or fuller upper eyelids, ${ }^{15}$ which prevent the attainment of a high upper eyelid position during ptosis surgery.

A previous study recommended $1 \mathrm{~mm}$ of overcorrection during intraoperative adjustment when aponeurotic repair was performed using lidocaine with epinephrine. ${ }^{16}$ However, the $\triangle \mathrm{MRD}-1$ was significantly different between the groups (Group A, $0.68 \mathrm{~mm}$; Group $B, 0.17 \mathrm{~mm}$ ) in the present study. The application of $1 \mathrm{~mm}$ of overcorrection therefore carries a risk of creating an overcorrected upper eyelid position after aponeurotic repair in patients with a negative response to the phenylephrine test. Hence, surgeons need to apply intraoperative overcorrections based on the results of the phenylephrine test.

The distribution of local anaesthetic after injection into the eyelid is unknown. However, as the orbital septum is an inelastic, multilaminar, fibrous sheet, ${ }^{11}$ it may block deep infiltration of local anaesthetic toward the Müller muscle. The attachment site of the orbital septum to the levator aponeurosis was thought to extend inferiorly to the tarsal plate in Japanese patients, ${ }_{17}$ suggesting complete blockage of any deep infiltration. However, the orbital septum reportedly attaches to the levator aponeurosis above the tarsal plate, even in Japanese patients ${ }^{15}$ which implies that local anaesthetics can infiltrate the Müller muscle at least between the upper edge of the tarsal plate (distal top of the Müller muscle) and the attachment site of the orbital septum. In this situation, both epinephrine and lidocaine infiltrates into the Müller muscle. However, we can intraoperatively 
obtain elevation of the upper eyelid with MMCR, indicating that the Müller's muscle can contract due to the epinephrine without severe paresis being caused by the lidocaine.

A previous study reported another technique that included simultaneous resection of the infiltrated part of the Müller muscle to eliminate the effect of sympathetic nerve stimulation on the Müller muscle. ${ }^{18,19}$ As the levator aponeurosis was solely advanced in the present study, the results may not be applicable to the previously reported technique. Further studies are necessary to determine the correlation between the results of the phenylephrine test and the $\triangle \mathrm{MRD}-1$ after the previously reported technique.

Local anaesthetics can infiltrate the levator palpebrae superioris muscle during blepharoptosis surgery. ${ }^{20}$ In such a situation, a large amount of advancement of the levator aponeurosis is necessary for obtaining the appropriate intraoperative MRD-1 from an excessively lowered upper eyelid position caused by the paralytic levator palpebrae muscle. This step causes postoperative overcorrection after restoring the function of the levator palpebrae superioris muscle. ${ }^{20}$ However, none of the patients in the present study exhibited an intraoperative eyelid droop or postoperative overcorrection, suggesting that the local anaesthetic had little or no effect on the levator palpebrae superioris muscle.

Local anaesthetic injection paralyses the orbicularis oculi muscle. As this muscle is an antagonist of the levator aponeurosis, ${ }^{21}$ paralysis of the orbicularis oculi may cause intraoperative elevation of the upper eyelid. However, the volume of local anaesthetic used for injection did not differ between the groups, suggesting that this would have had little influence on the present results.

The present study found that 24 of 40 patients (60.0\%) had a negative phenylephrine test result. This percentage is higher than that reported in the UK. ${ }^{22,23}$ One of the possible reasons is the presence of a fuller upper eyelid due to downward extension of the preaponeurotic fat pad in Japanese patients. ${ }^{15}$ This heavy upper eyelid may prevent rising of the upper eyelid after application of phenylephrine eye drops.

Our study was limited by several factors. First, this study had a retrospective design and comprised a relatively small sample size. A larger number of patients would provide a greater statistical power. Another limitation was the inclusion of only Japanese patients. The results of the present study may not be applicable to other nationalities. We chose transcutaneous aponeurotic repair, not MMCR, in patients with a positive phenylephrine response; this was done to avoid corneal abrasion and to allow simultaneous creation of the eyelid crease that is absent in some Japanese patients. ${ }^{24}$

However, as the present results may not be applicable to
MMCR, future studies are needed to confirm the correlation in MMCR. Finally, only 5\% phenylephrine eye drops are commercially available in Japan, although $2.5 \%$ phenylephrine eye drops are commonly used in other countries. ${ }^{25,26}$ A previous study showed more cardiovascular adverse effects after $10 \%$ phenylephrine instillation, compared with $2.5 \%$ phenylephrine instillation. ${ }^{27,28}$ Although we used 5\% phenylephrine eye drops, this may cause a greater risk of adverse side effects than $2.5 \%$ phenylephrine instillation. On the contrary, although another study demonstrated greater elevation of ptotic upper eyelids after $10 \%$ phenylephrine instillation than after $2.5 \%$ phenylephrine, the difference in eyelid elevation was quite small. ${ }^{29}$ The disparity between 2.5 and $5 \%$ concentrations may, therefore, not produce a large difference in eyelid elevation after phenylephrine instillation.

In conclusion, the $\triangle \mathrm{MRD}-1$ was considerable in Group A but was clinically negligible in Group B. In addition, a significantly moderately positive correlation was found between the $\triangle$ MRD-1 and the results of the phenylephrine test in the total patient group. These results indicate that the degree of a postoperative eyelid droop can be estimated by the results of the phenylephrine test in transcutaneous aponeurotic repair.

\section{Summary}

What was known before

- It is essential for oculoplastic surgeons to identify factors affecting postoperative eyelid height. Epinephrinecontaining local anaesthetic stimulates the Müller muscle, it occasionally results in an undercorrected upper eyelid position after loss of the epinephrine effect. However, the frequency and degree of the upper eyelid droop after loss of the epinephrine effect has not been examined.

What this study adds

- There was a moderate positive correlation between phenylephrine response and the $\Delta \mathrm{MRD}-1$, which is defined as the difference between the postoperative MRD-1 value and the intraoperative value. The degree of a postoperative eyelid droop can be estimated by the results of the phenylephrine test in transcutaneous aponeurotic repair.

\section{Conflict of interest}

The authors declare no conflict of interest.

\section{References}

1 Waqar S, McMurray C, Madge SN. Transcutaneous blepharoptosis surgery-advancement of levator aponeurosis. Open Ophthalmol J 2010; 14: 76-80. 
2 Scoppettuolo E, Chadha V, Bunce C, Olver JM, Wright M BOPSS. British Oculoplastic Surgery Society (BOPSS) National Ptosis Survey. Br J Ophthalmol 2008; 92: 1134-1138.

3 McCulley TJ, Kersten RC, Kulwin DR, Feuer WJ. Outcome and influencing factors of external levator palpebrae superioris aponeurosis advancement for blepharoptosis. Ophthal Plast Reconstr Surg 2003; 19: 388-393.

4 Mauriello Jr JA, Abdelsalam A. Modified levator aponeurotic advancement with delayed postoperative office revision. Ophthal Plast Reconstr Surg 1998; 14: 266-270.

5 Aghai GH, Vazirnia M, Poormatin R, Falavarjani KG. Effect of local anesthesia with bupivacaine plus epinephrine on blepharoptosis and levator palpebrae muscle function. Ophthal Plast Reconstr Surg 2013; 29: 198-200.

6 Bartley GB, Lowry JC, Hodge DO. Results of levatoradvancement blepharoptosis repair using a standard protocol: effect of epinephrine-induced eyelid position change. Trans Am Ophthalmol Soc 1996; 94: 165-173.

7 Skibell BC, Harvey JH, Oestreicher JH, Howarth D, Gibbs A, Weqrynowski $\mathrm{T}$ et al. Adrenergic receptors in the ptotic human eyelid: correlation with phenylephrine testing and surgical success in ptosis repair. Ophthal Plast Reconstr Surg 2007; 23: 367-371.

8 Ben Simon GJ, Lee S, Schwarcz RM, McCann JD, Goldberg RA. Muller's muscle-conjunctival resection for correction of upper eyelid ptosis: relationship between phenylephrine testing and the amount of tissue resected with final eyelid position. Arch Facial Plast Surg 2007; 9: 413-417.

9 Boboridis K, Assi A, Indar A, Bunce C, Tyers AG. Repeatability and reproducibility of upper eyelid measurements. Br J Ophthalmol 2001; 85: 99-101.

10 Baldwin HC, Bhaqey J, Khooshabeh R. Open sky Müller muscle-conjunctival resection in phenylephrine test-negative blepharoptosis patients. Ophthal Plast Reconstr Surg 2005; 21: 276-280.

11 Kakizaki H, Malhotra R, Selva D. Upper eyelid anatomy: an update. Ann Plast Surg 2009; 63: 336-343.

12 Federici TJ, Meyer DR, Lininger LL. Correlation of the vision-related functional impairment associated with blepharoptosis and the impact of blepharoptosis surgery. Ophthalmology 1999; 106: 1705-1712.

13 Kakizaki H, Kinoshita S. Ptosis surgery: Muyamini Kinwo Agerubekarazu. Jpn J Ophthalmic Surg 2007; 20: 404 (Japanese).

14 Watanabe A, Kakizaki H, Selva D, Ohmae M, Yokoi N, Wakimasu $\mathrm{K}$ et al. Short-term changes in tear volume after blepharoptosis repair. Cornea 2014; 33: 14-17.

15 Kakizaki H, Leibovitch I, Selva D, Asamoto K, Nakano T. Orbital septum attachment on the levator aponeurosis in Asians: in vivo and cadaver study. Ophthalmology 2009; 116: 2031-2035.
16 Anderson RL, Dixon RS. Aponeurotic ptosis surgery. Arch Ophthalmol 1979; 97: 1123-1128.

17 Jeong S, Lemke BN, Dortzbach RK, Park YG, Kang HK. The Asian upper eyelid: an anatomical study with comparison to the Caucasian eyelid. Arch Ophthalmol 1999; 117: 907-912.

18 Noma K, Takahashi Y, Leibovitch I, Kakizaki H. Transcutaneous blepharoptosis surgery: simultaneous advancement of the levator aponeurosis and Müller's muscle (levator resection). Open Ophthalmol J 2010; 14: 71-75.

19 Takahashi Y, Kakizaki H, Mito H, Shiraki K. Assessment of the predictive value of intraoperative eyelid height measurements in sitting and supine positions during blepharoptosis repair. Ophthal Plast Reconstr Surg 2007; 23: 119-121.

20 Ismail AR, Anthony T, Mordant DJ, MacLean H. Regional nerve block of the upper eyelid in oculoplastic surgery. Eur J Ophthalmol 2006; 16: 509-513.

21 Janfaza P, Cheney ML. 2011. Superficial structures of the face, head, and parotid region. In: Janfaza P, Nadol Jr JB, Galla RJ, Fabian RL (eds). Montgomery WWSurgical Anatomy of the Head and Neck. Harvard University Press: Cambridge, MA, USA, 2011, pp 11.

22 Malhotra R, Salam A. Outcomes of adult aponeurotic ptosis repair under general anaesthesia by a posterior approach white-line levator advancement. Orbit 2012; 31: 7-12.

23 Al-Abbadi Z, Saqili S, Malhotra R. Outcomes of posteriorapproach 'levatorpexy' in congenital ptosis repair. Br J Ophthalmol 2014; 98: 1686-1690.

24 Kakizaki H, Takahashi Y, Nakano T, Asamoto K, Ikeda H, Iwaki $\mathrm{M}$ et al. The causative factors or characteristics of the Asian double eyelid: an anatomic study. Ophthal Plast Reconstr Surg 2012; 28: 376-381.

25 Mota PM, Norris JH. Review on surgical management of ptosis and the use of phenylephrine: a national survey of British Oculoplastic Surgery Society (BOPSS) UK Consultants. Orbit 2016; 6: 1-4.

26 Samimi DB, Erb MH, Lane CJ, Dresner SC. The modified fasanella-servat procedure: description and quantified analysis. Ophthal Plast Reconstr Surg 2013; 29: 30-34.

27 Stavert B, McGuinness MB, Harper CA, Guymer RH, Finger RP. Cardiovascular adverse effects of phenylephrine eyedrops: a systematic review and meta-analysis. JAMA Ophthalmol 2015; 133: 647-652.

28 Suwan-Apichon O, Ratanapakorn T, Panjaphonqse R, Sinawat S, Sanguansak T, Yospaiboon Y. 2.5 and $10 \%$ phenylephrine for mydriasis in diabetic patients with darkly pigmented irides. J Med Assoc Thai 2010; 93: 467-473.

29 Glatt HJ, Fett DR, Putterman AM. Comparison of 2.5 and $10 \%$ phenylephrine in the elevation of upper eyelids with ptosis. Ophthalmic Surg 1990; 21: 173-176. 